\title{
Neuronal Ceroid Lipofuscinosis Type 3
}

National Cancer Institute

\section{Source}

National Cancer Institute. Neuronal Ceroid Lipofuscinosis Type 3. NCI Thesaurus. Code C61258.

A condition associated with mutation(s) in the CLN3 gene, encoding battenin. The condition is one of a group of genetically heterogeneous neurodegenerative disorders, characterized by accumulation of intracellular lipopigments. 